\title{
Myomatous Neoplasm
}

National Cancer Institute

\section{Source}

National Cancer Institute. Myomatous Neoplasm. NCI Thesaurus. Code C4063.

A benign or malignant mesenchymal neoplasm arising from smooth, skeletal, or cardiac muscle. 